\title{
The study of dynamic interest changes based on complex network
}

\author{
Bing $\mathrm{Li}^{1 \text {, a }}$, Hui Zheng ${ }^{1}$ and Yanjun Gui ${ }^{2}$ \\ ${ }^{1}$ Wuhan University of Technology, Wuhan, Hubei, China \\ ${ }^{2}$ NTS Automobile Service Co. Ltd, Shanghai, China \\ ae-mail:lib675@whut.edu.cn
}

\begin{abstract}
It is the most important way to improve recommendation precise by catching the interests of users. It is easy to find current interests of users; however, a good recommendation should provide potential interests to uses. In this work, the inner and outer factors are combined to analyze the changes of interests. The inner factor addresses the changes from the innate characteristics of people relevant with interests. The friendship in the social network as the outer factor plays role in generating novel interests. Since the work exactly describes the changes of interests; it is useful to improve the satisfaction of user to recommendation.

Keywords: Dynamic interest; complex network; inner and outer factors; link prediction.
\end{abstract}

\section{Introduction}

The precise of recommendation becomes the most important factor deciding the success of E-business [1]. A good recommendation system should understand what provides the satisfaction to user. Now, most recommendation systems focus on the interesting of customers [2,3]. However, the interests of users do not keep the same. Thus, it is very important to know the ruless affecting the changes of interests.

Link Prediction in complex network is used to research the changes of interests. First, the performance of Link Prediction of complex network has been verified in the field of personal recommendation systems [4, 5]. Second, social network goes deep into every corner of people. A large amount of information exchanges are 
carried out in everyday. As a result, the information exchanges in the social network affect the interests of users. Social network is a novel, interesting, and typical complex network.

In recommendation systems, Link Prediction is used to find potential friendship; then, one node will receive a massage which describes the potential friends related to the node [4]. Classical Link Prediction gets the conclusion by employing Markov Chain. This kind of methods considers the characteristics of nods to obtain well performance; however, the computational cost is high, and perfect parameter is hard to find. Probabilistic Relational Model [6] and Directed Acyclic Probabilistic Relational Model [7] can also be used in Link Prediction methods, which use different format to describe the objects. Among the methods of Link Prediction, it can be concluded by the experiments of real networks that those based on graph theory have very well performance. Those methods produce different indicators employing the similarity of network topology [8].

We find that the factors playing role in the changes of interests are not from the same directions. First, the variation of the own characteristic of users affects their interests, which has been discussed in our last work [9]. Since there are so many observed characteristics of people; a tool is necessary to give the exact ones related to the interests. In that work, we proposed a novel feature selection method, RSMI-FS with multi-labels, to find the set of characteristics reflecting the interests. Then, according to the clustering algorithm SOM, the similar variation of interests among people is described. That is to say that the interests coming from inner characteristics had been expressed. However, it is just one aspect playing role in the changes of interest. Social network has been an important communication way. When one person receives a topic belonging to a novel interest, it is possible to take this new interest. In this work, we define social network is the outer factor of dynamic interests. In order to combine the inner and outer factors, we firstly build a network based on social network. Then, interests and their relation with the person nodes are inserted into the network to generate a complete description 
system. At last, Link Prediction is employed to foresee the future interests of person nodes.

The rest of paper is organized as follows. Section 2 gives the generation of initial network. The brief content of inner factor affecting the changes of interests is in Section 3. The process of adding novel interests using the idea of Link Prediction is presented in Section 4. Finally, the conclusion is drawn in Section 5.

\section{Generation of Initial Network}

An initial network can be built based on the real social network as following:

- Every person is set as a node of the initial network;

- If person $\mathrm{A}$ follows person $\mathrm{B}$ or person $\mathrm{A}$ is a friend of person $\mathrm{B}$, there is a direct link from $\mathrm{A}$ to $\mathrm{B}$.

The example graph in Fig. 2.1(a) is translated into the network in Fig. 2.1(b) as this method. Every circle denotes a person in the social network; the edge describes the relationship, and arrow goes from node $\mathrm{A}$ to node $\mathrm{B}$ when person $\mathrm{A}$ follows person B. For node A and node B, there are three kinds of friendships explained in Fig. 2.2. Then, interests expressed by bucket are inserted in the network. If a person is interested in one topic, an edge will be added between the person node and the related interest. The complete network is shown in Fig. 2.3.

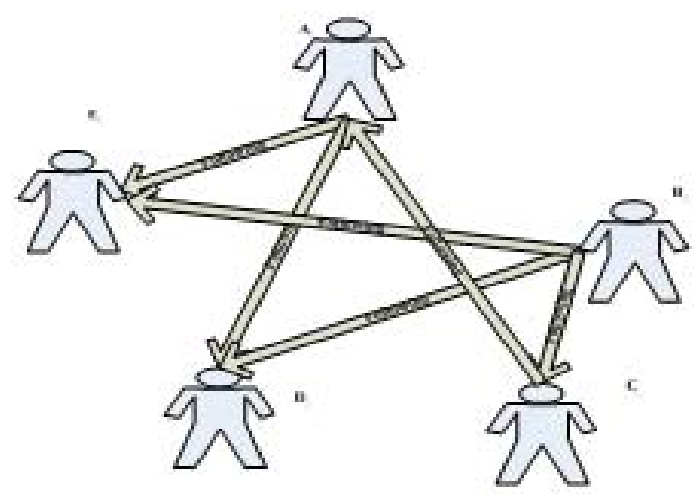

(a) 


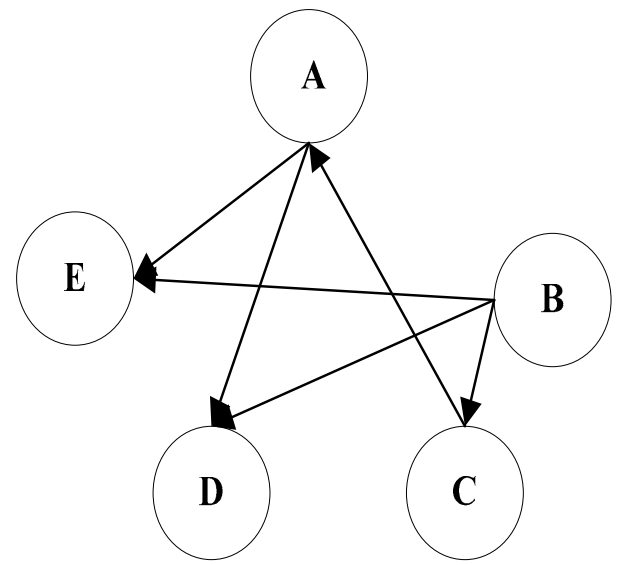

(b)

Fig.2.1 Translation of social network. (a) real social network; (b) simulated network.

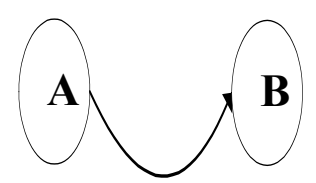

(a)

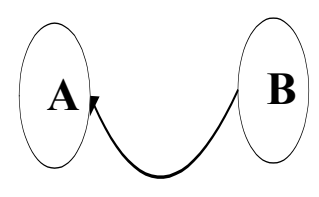

(b)

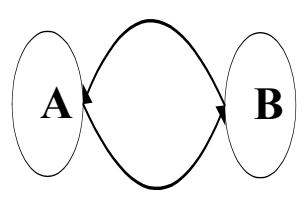

(c)

Fig.2.2 Friendship of nodes.

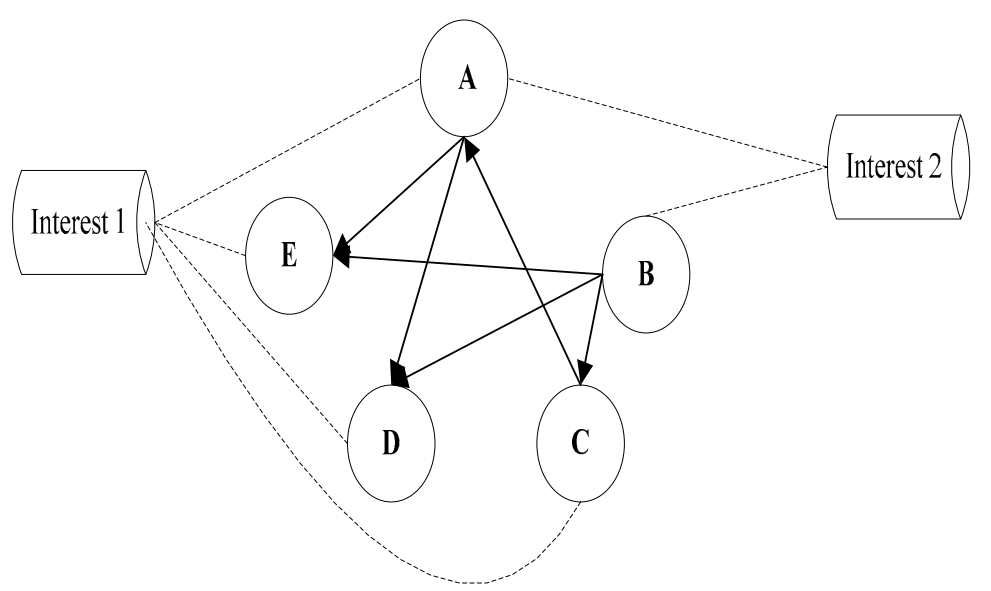

Fig. 2.3 Complete network. 


\section{Inner factors of dynamic network}

Innate characteristics of people are the most direct factor affecting the changes of interests, so we discuss the complete mechanism of the inner factors in [9]. However, inner factor is just one aspect in the process of dynamic interests. Thus, in this work, the outer and inner factors are combined to research the changes of interest. In order to shown complete theory, a brief description of inner factor is shown.

The dynamic interest is very special application, where there are more than one label features. Current feature selection method cannot deal with this question. A novel feature selection method, RSMI-FS with multi-labels, is proposed to mine the relevant characteristics with interests. After that, clustering algorithm estimates the similarity among the people with temporal interests and a stranger to obtain the future interests of the stranger.

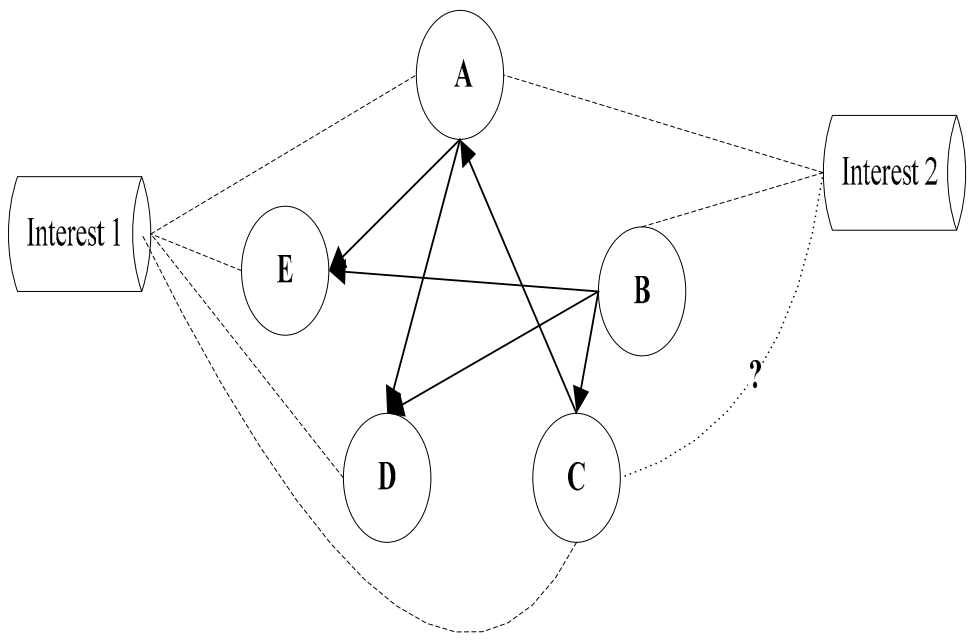

Fig. 4.1 Result of Like Prediction.

Methodology 3.1 Add novel interests of a user node by link prediction

Case 1) Check the time trigger. If a new time slice starts,

I. Get the rank of the possible interests according to the result of Algorithm Getting Future Interests[9].

II. Add a future-link between user node-and the related interest-node.

Case 2) Node A and node B are both user node. Node A send a message. 


\begin{tabular}{ll}
\hline I. & $\begin{array}{l}\text { Collect the interests of node B. And Judge whether the content } \\
\text { belongs to the set of interests. If it is not a new interest, break; } \\
\text { otherwise, locate the new interest. }\end{array}$ \\
II. & Check the friendship of A and B. If they follow each other, go to \\
III of Case 2; If B follows A, go to IV of Case2; otherwise, break. \\
Add a future-link between B and the interest with the possibility \\
of $\theta$.
\end{tabular}

\section{Add Novel Interests Using Link Prediction}

The communication style has been both on-line and off-line, and the user has put more and more attention to on-line, especially social network. This is the outer factor affecting the interests. According to the analysis in Fig. 2.2, we know that there are three kinds of friendship in social network. It is reasonable that the friendship is the strongest when two nodes follow each other. In this case, the acceptance of a new interest recommended by his friend is easier. If node A follows $\mathrm{B}$, we can believe that $\mathrm{A}$ is very interested in the content from $\mathrm{B}$. It is possible that A accept the new interest from B. However, it is very rare that B get the information from A.

On the basis of the inner and outer factors, the networks of persons and interests can be reconstructs. Methodology 3.1 shows the mechanism of interest changes. Case 1 describes the mechanism of inner factors. In order to find the novel interests from inner factor, RSMI-FS with multi-labels is firstly used to decide the relevant characteristics with interests [9]. After that, the algorithm, Getting Future Interests, provides a rank of interests which has large possibility to be the future interests of a person node in the network. In this work, we use time slice to check the interest updating. The setting of time slice can be got by domain experts. Links between a person node and its possible interests are inserted into the network according to the idea of Link Prediction. Beside the inner factors, the above analysis of social network is shown in the Methodology 3.1. Since the close levels of friendship are different; the message including a novel topic plays different role in the generating a new interest. It is reasonable to add a new link between a 
person node and an interest node with variable possibility which is decided by the close level of friendship.

Here, we import future link between the user node and the interest node. That is because that, in the current state, the user just receives the novel interest. It is different from the real interests of the user, so we use a new type link to predict this, showing in Fig. 4.1. A new link between node C and node Interest 2 is inserted into the network according to the rule of additional interests. Thus, the link is expressed with question make to denote it is a possible relationship which need be verified.

\section{Conclusion}

In this work, a network of uses and interest is built to analyze the factors affecting the changes of interests. The topology of the network describes the complex relation among persons and interests. Using Link Prediction, the topology is revised to provide reasonable option for recommendation system. The basis of Link Prediction is the innate characteristics relevant with the interests and information exchanges in social network.

\section{Acknowledgement}

This work is supported by "the Fundamental Research Funds for the Central Universities (WUT: 2014-IV-026)" and "The National Scientific and Technological Support Project (2013BAH13F01)".

\section{References}

[1] J. A. Konstan, J Riedl, Recommender systems: from algorithms to user experience. User Modeling and User-adapted Interaction, Vol.22(2012), p.101-123.

[2] M Gao, Zhong Fu, F Jiang, Userrank for item-based collaborative filtering recommendation, Information Processing Letters, Vol,111(2011), p.440-446. 
[3] S. J. Yu,The dynamic competitive recommendation algorithm in social network services, information Science, Vol.187 (2012),p1-14.

[4]L. Schafer, J. W. Graham, Missing data: our view of the state of the art. Psychol Methods, Vol.7(2) (2002), p.147-177.

[5] G. Kossinets, Effects of missing data in social networks, Social Networks, Vol. 28(3) (2006),p, 247-268.

[6] P. H. Wuillemin, L Torti, Structured probabilistic inference, International Journal of Approximate Reasoning, Vol.53(7)(2012),p. 946-968.

[7] H. Li, D. Jiang, New model and heuristics for safety stock placement in general acyclic supply chain networks, Computers \& Operations Research, Vol.39(7)(2012), p.1333-1344.

[8] E. A. Leicht, P. Holme, M. E. J. Newman, Vertex similarity in networks, Physical Review E,Vol. 3(2)(2006), 026120.

[9] B. Li, H. Zheng, Y. Gui, The study of dynamic interest changes based on feature selection, submitted. 